\title{
Makeup Activates Brain Activity in Visually Impaired Persons: Evaluation by Functional Magnetic Resonance Imaging
}

\author{
Katsushi Taomoto ${ }^{*}$, Kaho Oishi' ${ }^{2}$, Hiroshi Matsunaka ${ }^{3}$, Yumi Murakami ${ }^{3}$, Yoshihiro Kuga1, \\ Shinsuke Hashimoto ${ }^{1}$, Hideyuki Ohnishi ${ }^{1}$, Koji Abe ${ }^{4}$ \\ ${ }^{1}$ Ohnishi Neurological Center, Akashi, Japan \\ ${ }^{2}$ International Caremake Association, Tokyo, Japan \\ ${ }^{3}$ NOV Academic Research, TOKIWA Pharmaceutical Co. Ltd., Tokyo, Japan \\ ${ }^{4}$ Department of Neurology, Graduate School of Medicine, Dentistry and Pharmaceutical Sciences, \\ Okayama University, Okayama, Japan \\ Email: *katsushi.taomoto@onc.akashi.hyogo.jp
}

How to cite this paper: Taomoto, K., Oishi, K., Matsunaka, H., Murakami, Y., Kuga, Y., Hashimoto, S., Ohnishi, H. and Abe, K. (2021) Makeup Activates Brain Activity in Visually Impaired Persons: Evaluation by Functional Magnetic Resonance Imaging. Journal of Cosmetics, Dermatological Sciences and Applications, 11, 140-154.

https://doi.org/10.4236/jcdsa.2021.112014

Received: March 10, 2021

Accepted: June 5, 2021

Published: June 8, 2021

Copyright ( 2021 by author(s) and Scientific Research Publishing Inc. This work is licensed under the Creative Commons Attribution International License (CC BY 4.0).

http://creativecommons.org/licenses/by/4.0/ (c) (i) Open Access

\begin{abstract}
Cosmetics are used to improve physical appearance, but the benefits may be limited to people without visual impairment. The importance of attractiveness among blind persons has not been assessed. We investigated the influence of makeup on brain activity of blind persons using functional magnetic resonance imaging (fMRI). Participants were 7 blind females (BFs) who learned to fully apply makeup and 9 mostly age-matched normally sighted females (NSFs). Brain activity was measured using fMRI before and after application of makeup and during a makeup image task in each state. In the default mode network at rest, there was no difference between the BFs and NSFs. However, a lateral visual network to the opposite side was observed in the NSFs, whereas no such network was noted in the BFs. A weak network was noted in the BFs in the occipital fusiform gyrus and temporal occipital fusiform cortex, and an extensive visual area network defect was noted. Also, activity after makeup application was significantly higher in the nucleus accumbens, pallidum, and hippocampus. Activity in the right middle cingulate gyrus, right cerebral white matter, and right anterior cingulate gyrus was higher before makeup in both BFs and NSFs, and the activity was significantly higher and more extensive in the BFs. In conclusion, applying makeup is a personally rewarding activity, even for BFs, as it strongly activates the reward system and the reward/memory system network, even in the absence of a visual area network.
\end{abstract}




\section{Keywords}

Functional Magnetic Resonance Imaging, Visually Impaired Persons, Brain Activity, Makeup, Female

\section{Introduction}

Cosmetics make us appear beautiful, but the benefits may be limited to sighted people. Makeup, including foundation and lipstick, is essential for the social life of females. Pretending to be sociable by being aware of oneself wearing makeup and conscious of being gazed at enables the mental improvement of individuals at work and in their daily life.

On the other hand, the importance of appearance among visually impaired persons has not been assessed because they cannot be aware of themselves wearing makeup or be conscious of being gazed at by others. However, visually impaired persons sense the reactions of others due to subtle changes in the words and atmosphere more so than the sighted persons. In addition, it has recently been reported that mental and behavioral changes due to applying makeup by visually impaired persons themselves render going out and meeting people fun [1]. Further, blind people experience emotions and carry out daily activities proactively based on the reactions of persons around them. The elucidation of brain function and pathology has rapidly progressed due to the development of computers and advances in statistical analysis methods, and functional magnetic resonance imaging (fMRI) is a highly reliable analytical method capable of visualizing changes in local brain blood flow accompanying nerve cell activity. In general, a task inducing nerve cell activity in the objective region was applied and observed, but studies on connectivity of the brain function at resting state MRI (rsfMRI) have recently markedly progressed because brain function can be analyzed without a task. In addition, many studies on the default mode network (DMN), especially those on cognitive function [2] [3] [4] and psycho-neurologic disease, have been published [5] [6] [7].

The visual sensation of sighted persons has been closely investigated using fMRI, and the regions of objects in the visual area and the recognition mechanism have been clarified [8] [9]. However, to the best of our knowledge, there has been no report on DMN in visually impaired persons. Thus, in this study, fMRI was used to analyze how cosmetic usage affects brain activity in blind people.

\section{Materials and Methods}

\subsection{Subjects}

The subjects were 7 blind females (BFs) who took a course and developed the ability to fully apply makeup by themselves, alongside 9 normally sighted females (NSFs) of the same or a similar age. 


\subsubsection{Selection Criteria}

- Applying makeup by oneself on a daily basis.

- Provision of written consent (verbal record was acceptable because of blindness) by free will after receiving an explanation of and understanding the study. When a disease other than skin disease was present, treatment was not restricted.

\subsubsection{Exclusion Criteria}

- Severe skin disease on the face.

- History of skin symptoms, such as contact dermatitis, due to the components of the test product and analogous components.

- Contraindications for fMRI measurements (such as gold thread embedded by cosmetic surgery).

- Use of drugs influencing brain activity.

- Pregnancy.

- Judged as ineligible to participate in this study by the physician in charge.

\subsection{Regulation of Concomitant Drugs (Therapy)}

Treatment of other diseases and complications being performed from before initiation of this study was continued. When a therapeutic drug (oral and external preparations) was used, the type, administration method, and dose were not changed during the study period as a general rule. However, when a drug influencing brain activity was used, the patient was excluded based on the judgment of the physician in charge.

\subsection{Approval by the Ethics Committee and Informed Consent}

The study protocol was approved by the ethics committee of Okayama University Faculty of Medicine and Ohnishi Neurological Center, and written consent was acquired from each normally sighted subject. For visually impaired persons, the physician in charge of the inspection institute verbally read the consent form and received oral consent from the subject.

\subsection{Evaluation}

\subsubsection{Procedure}

The following measurements were performed on the blind and control sighted persons using the SIGNA Architect 3.0-T MRI (General Electric Corp., Boston, MA, USA).

1) fMRI measurement in a resting state without makeup;

2) fMRI measurement during an image task of applying makeup without using cosmetics;

3) fMRI measurement in a resting state with makeup;

4) fMRI measurement after the image task of applying makeup using cosmetics;

5) Standard MRI (diffusion weighted imaging [DWI], T2, fluid attenuated inversion recovery [FLAIR], and $\left.\mathrm{T} 2^{\star}\right)$ acquisition. 
The cosmetics used were unscented, from the "NOV makeup series" (TOKIWA Pharmaceutical Co. Ltd., Japan). All compounds and raw materials met the criteria for cosmetic materials of "the Law for Ensuring the Quality, Efficacy, and Safety of Drugs and Medical Devices" and are commercially available. The time needed to apply makeup in the next room after measurement of 2) was approximately 15 minutes for both visually impaired and sighted persons, and they applied similar makeup styles using the cosmetics. To render the conditions of the NSFs uniform with those of the BFs, the NSFs wore an eye mask and closed their eyes during MRI scans.

\subsubsection{Oral History}

1) Age and presence of skin disease;

2) Dominant hand;

3) Frequency of use of makeup and items used;

4) The presence of tattoos or gold thread embedded by cosmetic surgery;

5) Conduction of fMRI in the past;

6) Age at onset of visual impairment (only for BFs).

\subsubsection{Questionnaire}

The questionnaire below was answered by all subjects and the score was summed. In the BFs, after the fMRI measurements, the physician in charge of the assessment orally questioned and recorded answers in the makeup room. The sighted persons filled out the survey by themselves.

1) Interest in MRI (very interested: 3, normal: 2, not very interested: 1 );

2) Anxiety about MRI (very strong: 3 , normal: 2 , almost none: 1 );

3) Sleepiness during test (strong: 4, slightly strong: 3, a little: 2, none: 1 );

4) Performance of makeup in test (very good: 5, good: 4, normal: 3, slightly poor: 2, poor: 1);

5) Advantages of makeup (Fun, happy, gaining confidence: 1 point each);

6) Specific effects of makeup (joy, confidence, smiling face, improvement of depression, relief of anxiety, improvement of sense of alienation, elimination of inferiority complex, self-love, motivation, positivity, participation in society, and cooperation with the society) (1 point each).

\subsubsection{MRI Measurement Methods}

1) Device used: SIGNA Architect 3.0-T MRI of GE, 48-channel head coil.

2) Acquisition conditions:

Morphological image: 3DT1WI, DWI, FLAIR, and T2WI;

Functional image at rest: Gradient echo planar imaging (EPI);

Frequency field of view (FOV): $21.2 \mathrm{~cm}$, TR: $2500 \mathrm{~ms}$, matrix size $64 \times 64$;

Phase FOV: 1.0, TE; $30 \mathrm{~ms}$, frequency encode: R/L;

Slice thickness: $3.2 \mathrm{~mm}$, flip angle: 80 , sensitivity correction: none, spacing: 0.8 $\mathrm{mm}$, adding frequency: 1, ASSET: $2.0 \mathrm{Ph}$;

Number of slices: 40, 180 samples, acquisition time: 7 min $40 \mathrm{~s}$. 
All participants were confirmed to be awake by technologists during all measurements, and head movement within $5^{\circ}$ was detected using a monitoring device.

\subsubsection{Brain Activity Analysis}

For the brain activity analysis, functional brain mapping was performed using SPM 12 (Wellcome Department of Cognitive Neurology, London).

First, movement in the functional images was corrected by preprocessing, and anatomical images (T1) were registered to functional images (MNI T1) and subjected to an affine conversion. After segmentation of anatomical images, normalization of functional images, spatial smoothing, and batch processing were performed.

Individual analysis of brain activity (first-level analysis) was incorporated into the general linear model (GLM) after the design matrix preparation. Partial regression coefficients of regressors of individual GLM were calculated, and the contrast between conditions was prepared, after which statistical tests were carried out.

For the population analysis of brain activity (second-level analysis), the design matrix was prepared, after which a statistical estimation was carried out.

\subsubsection{Neural Network Analysis}

For the functional connectivity analysis, CONN toolbox release 17

(https://www.nitrc.org/projects/conn, RRID: SCR_009550) software was used. Fundamental information was input during setup, after which the structural images and functional images of the brain were set, and preprocessing (correction, registration, segmentation, normalization, and smoothing) was performed. Regions of interest (ROIs) were then set, and primary covariates (covariates level) and secondary covariates (covariates second-level) were set. Denoising processing, individual analysis of functional connectivity (first-level analyses), and ROI-to-ROI and seed-to-voxel analyses were performed as population analyses (second-level analyses).

For analytical images, DICOM images were converted to NIFTI images and analyzed using an exclusive personal computer. Numerical analysis was performed using Matlab (MathWorks ${ }^{\oplus}$, Natick, MA, USA).

\section{Results}

Examinations were carried out without any problem for all subjects. A total of 1.5 hours were required per person on average. Analyses were performed after completion of the test in all subjects.

\subsection{Background of Subjects}

There were $7 \mathrm{BFs}$ aged 30- to 73-year-old, and the mean age was 52-year-old. The causative diseases of visual impairment varied, and included retinoblastoma, eye abnormality, acute outer retinopathy, retinal pigmentary degeneration, congenital glaucoma, and congenital retinal pigmentary degeneration. Regarding the 
time to blindness onset, one subject was blind from immediately after birth and the other subjects transitioned from having low vision to blindness, with the duration of time prior to blindness onset ranging from 4 to 70 years, with a mean duration of 19.4 years, thereby demonstrating strong individual variation. Four subjects had jobs (one was on leave) and 3 were housewives. There were 9 NSFs aged 32- to 73-year-old, and the mean age was 55-year-old. All had jobs and were mainly medical workers. Two BFs were left-handed, but all NSFs were righthanded (Table 1).

\subsection{Questionnaire}

Of the questionnaire items, almost no difference was noted between the BFs and NSFs in terms of interest in makeup, anxiety for the test, sleepiness during the test, or impression of makeup. Regarding the advantages and effects of makeup, the BFs strongly felt the effects of makeup (Figure 1).

\subsection{Individual Analysis}

Optic nerve atrophy was noted in all BFs on conventional MRI, but there was almost no change in the brain parenchyma, and only a change considered to be an old infarction was observed in the left occipital lobe medial secondary visual area (BM 18) in a 70-year-old subject with congenital blindness. Among the NSFs, no abnormal finding was noted in the brain in any subject.

The active regions on fMRI scans at rest and during the image task varied among the BFs and NSFs, but no specific tendency was noted. In the congenitally blind subject, activity was noted only in the frontal lobe in contrast to the extensive activity noted in the parietal and temporal lobes rather than the frontal lobe in the other subjects.

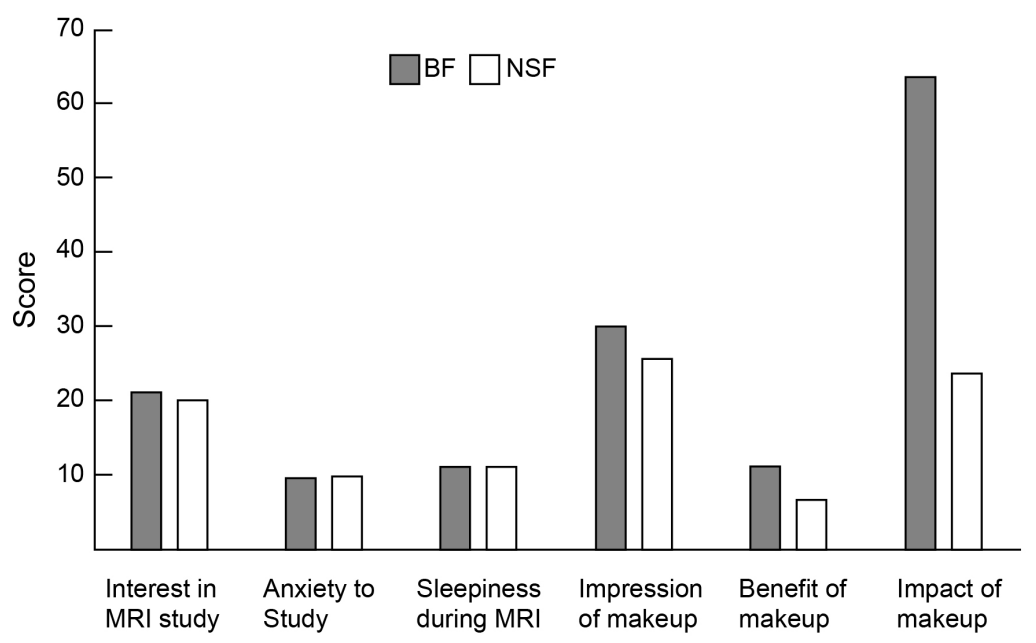

Figure 1. Results of the questionnaire between BF and NSF. There were no differences in the responses to questions regarding the MRI study, anxiety to study, sleepiness during MRI, or makeup impression. However, many BF responses reflect the benefit of makeup and impact of makeup compared to NSF. BF, blind female; NSF, normal sighted female; MRI, magnetic resonance imaging. 
Table 1. Participant demographics.

\begin{tabular}{|c|c|c|c|c|c|c|c|c|c|}
\hline No. & ID & Age & Occupation & $\begin{array}{l}\text { Dominant } \\
\text { hand }\end{array}$ & Cause of blindness & $\begin{array}{c}\text { Age at } \\
\text { blindness }\end{array}$ & $\begin{array}{l}\text { Duration of } \\
\text { blindness } \\
\text { [Years] }\end{array}$ & $\begin{array}{c}\text { Duration of } \\
\text { blind makeup } \\
\text { training } \\
\text { [Months] }\end{array}$ & $\begin{array}{c}\text { Makeup habit } \\
\text { before blind } \\
\text { makeup }\end{array}$ \\
\hline 1 & BF-1 & 73 & House wife & $\mathrm{R}$ & Retinitis pigmentosa & 55 & 18 & 10 & None \\
\hline 2 & BF-2 & 30 & Company worker & $\mathrm{L}$ & Binocular retinoblastoma & 26 & 4 & 6 & None \\
\hline 3 & BF-3 & 50 & Director of NPO & $\mathrm{R}$ & Retinitis pigmentosa & 39 & 11 & 5 & $\begin{array}{l}\text { Face powder } \\
\text { and lipstick }\end{array}$ \\
\hline 4 & $\mathrm{BF}-4$ & 64 & House wife & $\mathrm{R}$ & $\begin{array}{c}\text { Congenital } \\
\text { microphthalmia }\end{array}$ & 50 & 14 & 6 & $\begin{array}{l}\text { Foundation } \\
\text { and lipstick }\end{array}$ \\
\hline 5 & BF-5 & 41 & Nurse (on leave) & $\mathrm{R}$ & $\begin{array}{l}\text { Unknown (Acute } \\
\text { retinopathy suspected) }\end{array}$ & 37 & 4 & 12 & $\begin{array}{l}\text { Usual full } \\
\text { makeup }\end{array}$ \\
\hline 6 & BF-6 & 70 & House wife & $\mathrm{R}$ & Congenital glaucoma & 0 & 70 & 24 & $\begin{array}{l}\text { Skin lotion } \\
\text { and lipstick }\end{array}$ \\
\hline 7 & BF-7 & 38 & Trombonist & $\mathrm{L}$ & $\begin{array}{l}\text { Congenital malformation } \\
\text { of eyeball (R: blind, } \\
\text { L: amblyopia) }\end{array}$ & 23 & 15 & 6 & None \\
\hline 8 & NSF-1 & 64 & $\begin{array}{l}\text { Instructor of blind } \\
\text { makeup }\end{array}$ & $\mathrm{R}$ & & & & & \\
\hline 9 & NSF-2 & 72 & House wife & $\mathrm{R}$ & & & & & \\
\hline 10 & NSF-3 & 67 & Pharmacist & $\mathrm{R}$ & & & & & \\
\hline 11 & NSF-4 & 39 & Laboratory technician & $\mathrm{R}$ & & & & & \\
\hline 12 & NSF-5 & 73 & Pharmacist & $\mathrm{R}$ & & & & & \\
\hline 13 & NSF-6 & 32 & Office clerk & $\mathrm{R}$ & & & & & \\
\hline 14 & NSF-7 & 47 & Office clerk & $\mathrm{R}$ & & & & & \\
\hline 15 & NSF-8 & 56 & Nurse & $\mathrm{R}$ & & & & & \\
\hline 16 & NSF-9 & 50 & Nurse & $\mathrm{R}$ & & & & & \\
\hline
\end{tabular}

The ages at blindness describe the onset of blindness, which is defined as an absence of vision with, at most, minimal light perception. BF, blind female; NS, normal sighted; R, right; L, left; NPO, nonprofit organization.

\subsection{Population Analysis (Seed-to-Voxel Analysis)}

The functional connectivity at rest was analyzed in the 8 main brain networks in the BFs and NSFs (Table 2).

\subsection{Before and After the Makeup Task}

The anatomically activated regions before and after the makeup task were subjected to a subtraction analysis using SPM 12, and compared between the BFs and NSFs.

1) When connectivity of the DMN was observed, with the medial prefrontal cortex (MPFC), lateral prefrontal cortex (L, R: Left, Right), and posterior parietal cortex (PPC) as seeds, no difference was noted in any seed between the BFs and NSFs (Figure 2).

2) Regarding the lateral visual network, a network to the opposite visual area 
was clearly noted in both sides in the NSFs. In contrast, in the BFs, no network to the opposite side was observed (Figure 3), suggesting a lack of network to the primary visual area in the BFs.

3) In the occipital fusiform gyrus, similar to the lateral visual network, only weak activity to the opposite side was noted in the BFs, but activity was clearly noted in the NSFs (Figure 4).

Table 2. Major brain resting-state network.

\begin{tabular}{|c|c|c|}
\hline \multirow{3}{*}{1} & \multirow{3}{*}{ DMN (default mode network) } & MPFC (medial prefrontal cortex) \\
\hline & & LP (lateral prefrontal cortex) \\
\hline & & PPC (posterior parietal cortex) \\
\hline 2 & Sensory-motor & Lateral (L/R), Superior \\
\hline 3 & Visual & Medial, occipital, lateral (L/R) \\
\hline \multirow{3}{*}{4} & \multirow{3}{*}{ Salience } & ACC (anterior cingulate cortex) \\
\hline & & RPFC (rostral prefrontal cortex) (L/R) \\
\hline & & SMG (supramarginal gyrus) (L/R) \\
\hline \multirow{2}{*}{5} & \multirow{2}{*}{ Dorsal attention } & FEF (frontal eye field) (L/R) \\
\hline & & IPS (inferior parietal sulcus) (L/R) \\
\hline \multirow{2}{*}{6} & \multirow{2}{*}{ Fronto-parietal } & LPFC (lateral prefrontal cortex) (L/R) \\
\hline & & PPC (posterior parietal cortex) (L/R) \\
\hline \multirow{2}{*}{7} & \multirow{2}{*}{ Language } & IFG (inferior frontal gyrus) (L/R) \\
\hline & & pSTG (superior temporal gyrus, posterior) (L/R) \\
\hline 8 & Cerebellar & Anterior, posterior \\
\hline
\end{tabular}

CONN fMRI: Gabriell Lab, McGovern Institute for Brain Research, MIT; L, left; R, right; a Reference: Whitfield-Gabrieli, S. and Nieto-Castanon, A. (2012) CONN: A Functional Connectivity Toolbox for Correlated and Anticorrelated Brain Networks. Brain Connect, 2, 125-141.

https://doi.org/10.1089/brain.2012.0073
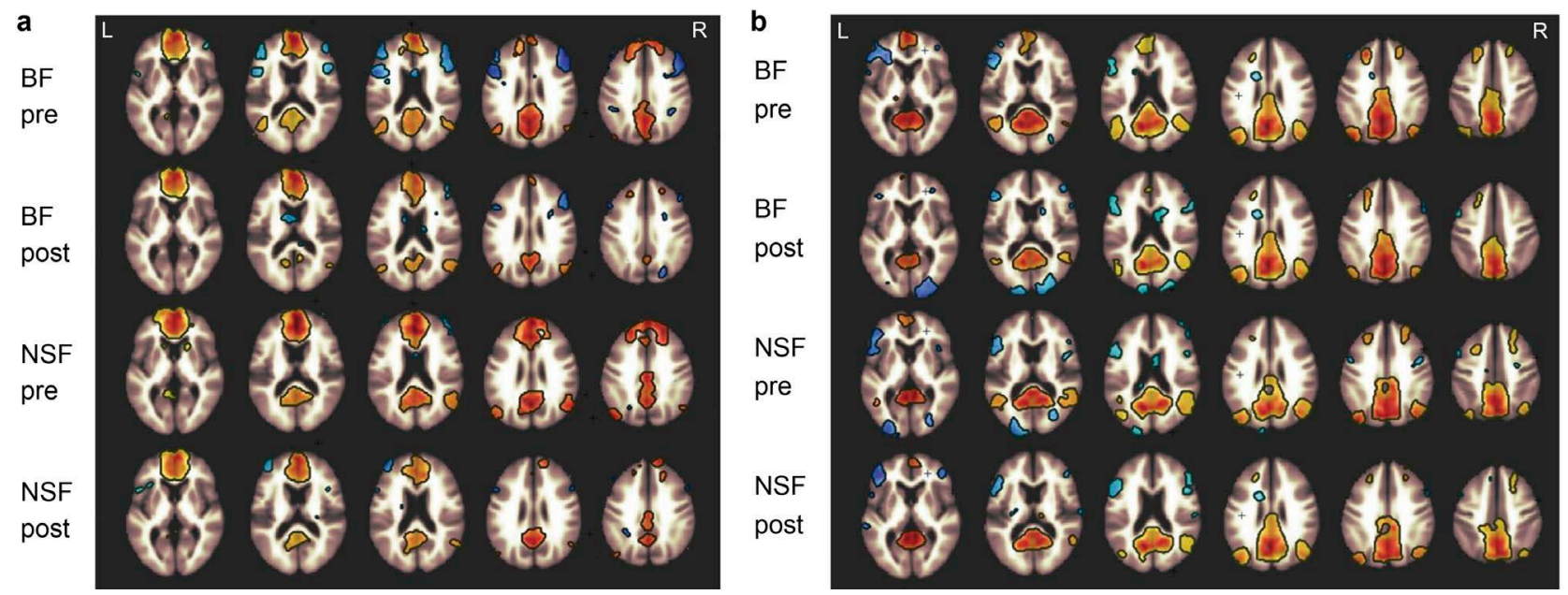

Figure 2. Connectivity of the default mode network. (a) Seed-to-Voxel analysis of the medical prefrontal cortex (MPFC). (b) Seed-to-Voxel analysis of the posterior cingulate cortex/precuneus (PCC). These results show no differences in default mode network activity between BF and NSF before and after makeup. L, left; R, right; BF, blind female; NSF, normal sighted female. 

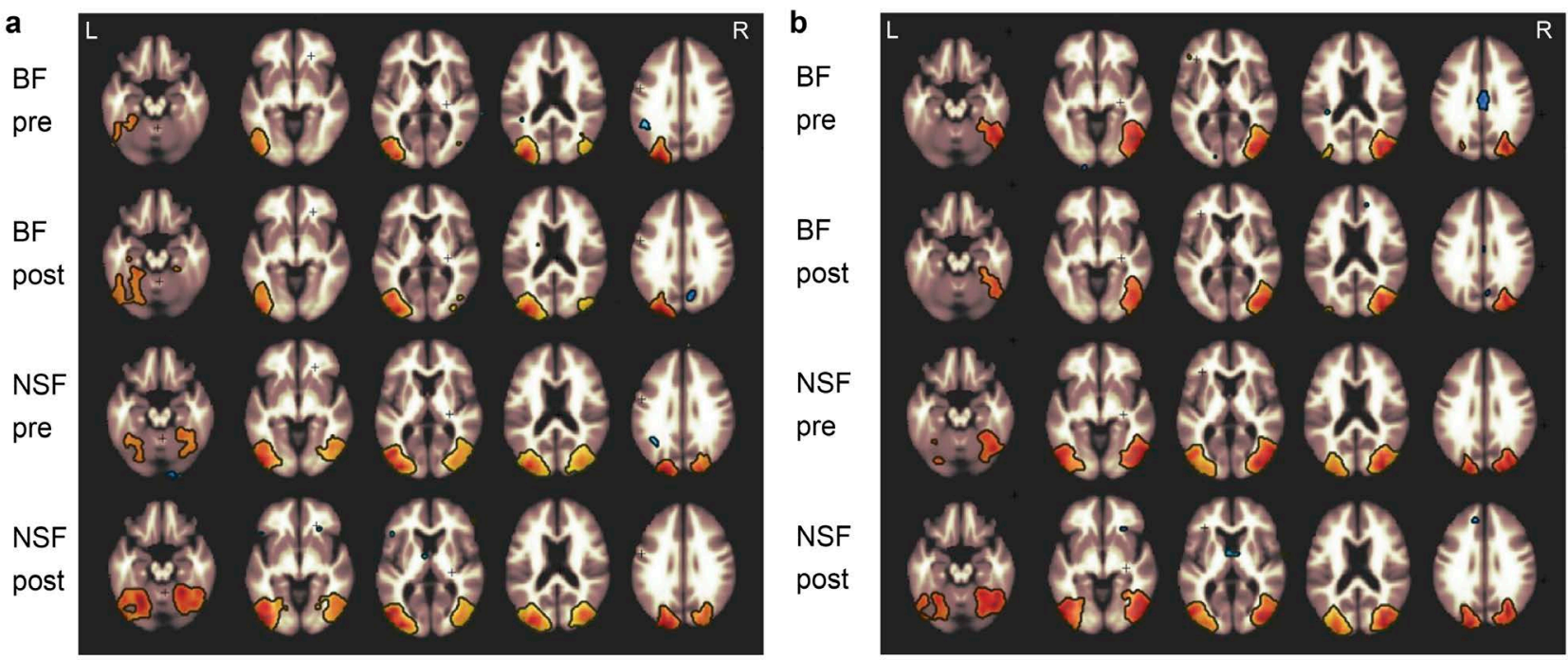

Figure 3. (a) Seed-to-voxel analysis of the lateral visual network (left). (b) Seed-to-voxel analysis of the lateral visual network (right). (a) Indicates no visual network from the lateral visual cortex to the opposite site, pre- and post-makeup in BF, while the NSF has a clear network between the right and left lateral visual cortex. (b) Shows that the seeds of the right visual cortex have no network to the left side in BF, but the NSF has a remarkable network between both the lateral visual cortex before and after makeup application. L, left; R, right; BF, blind female; NSF, normal sighted female.
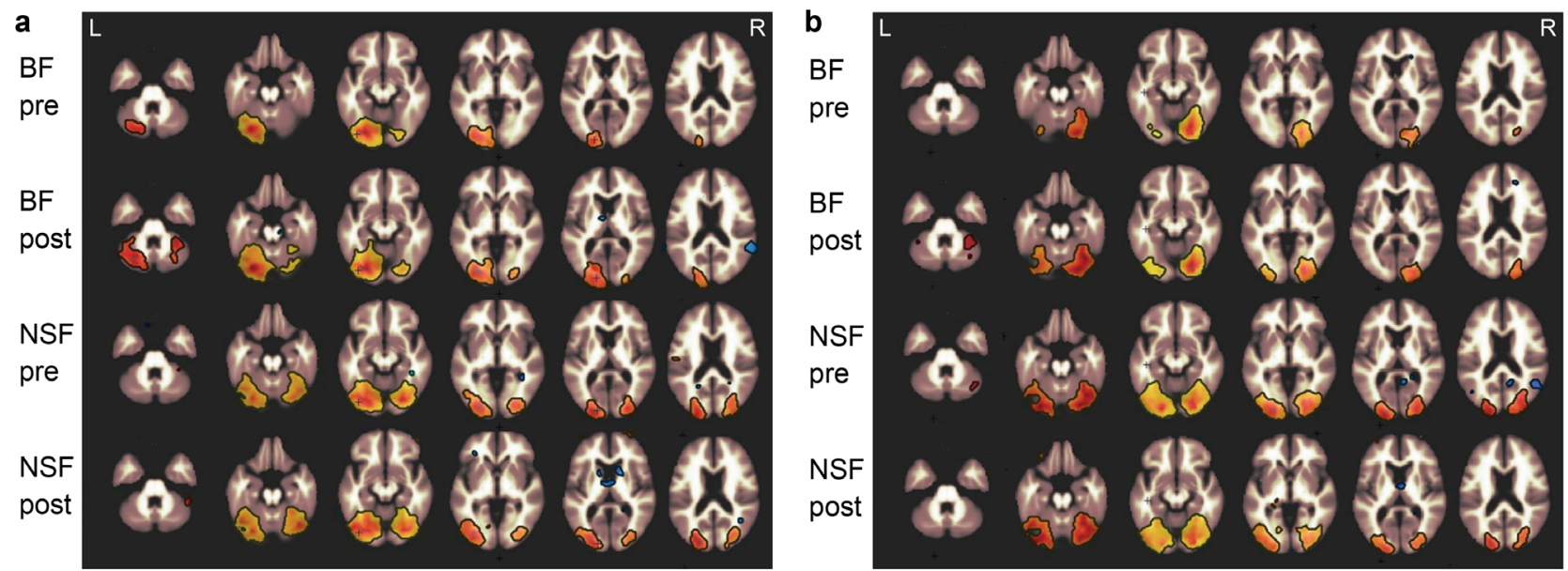

Figure 4. (a) Seed-to-voxel analysis of the occipital fusiform gyrus (left). (b) Seed-to-voxel analysis of the occipital fusiform gyrus (right). The occipital fusiform gyrus in both sides assumes the role of higher-level processing of visual information, including the identification and differentiation of objects, as well as being involved in memory, multisensory integration and perception. Both figures show the network differences of the occipital fusiform gyrus between BF and NSF. While the former group has no network between either side, the latter has a clear network between both sides. L, left; R, right; BF, blind female; NSF, normal sighted female.

4) In the temporal occipital fusiform cortex, only weak activity was noted on the opposite side in the BFs, but strong activity on the opposite side was clearly noted in the NSFs, suggesting that the network to the opposite side was absent or very weak in the secondary visual area and visual association area in the BFs.

5) Regarding the nucleus accumbens, the activity was significantly increased by makeup in the BFs compared with that in the NSFs, but no difference was noted in the network after makeup in the NSFs (Figure 5), suggesting that the 
joy experienced by makeup was stronger in the BFs than in NSFs because the nucleus accumbens is a nerve cell population of the reward system.

6) In the pallidum, the activity was stronger in the BFs than in NSFs and the reaction after makeup was stronger in the BFs, whereas it weakened in the NSFs (Figure 6).

7) In the hippocampus, activity was also slightly stronger in the BFs than in the NSFs.
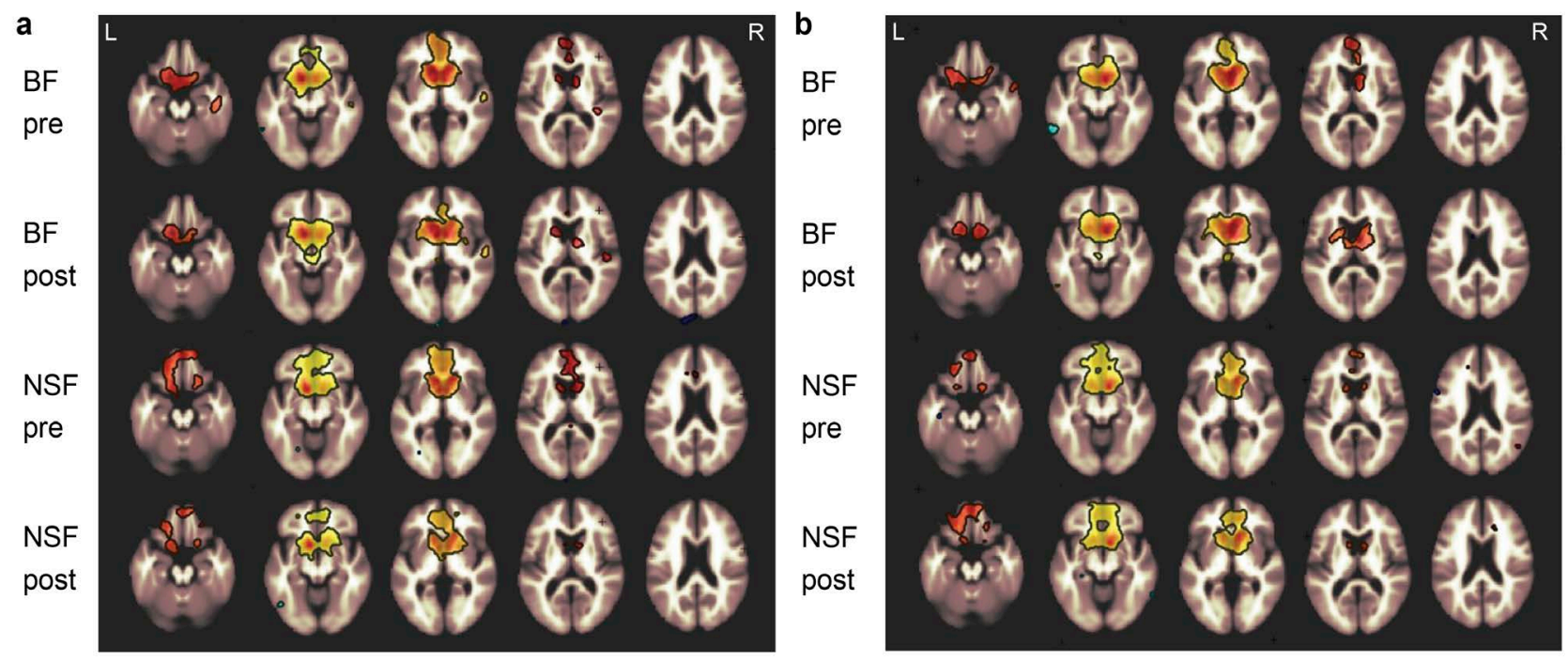

Figure 5. (a) Seed-to-voxel analysis of the accumbens (left). (b) Seed-to-voxel analysis of the accumbens (right). Response of both accumbens regions in BF was remarkably stronger than NSF. The accumbens area is considered a neural interface between motivation and action, playing a key-role in food intake, sexual behavior, reward-motivated behavior, stress-related behavior, and substance-dependence. The observed activity difference indicates that BFs may experience more joy on applying makeup than NSFs. L, left; R, right; BF, blind female; NSF, normal sighted female.
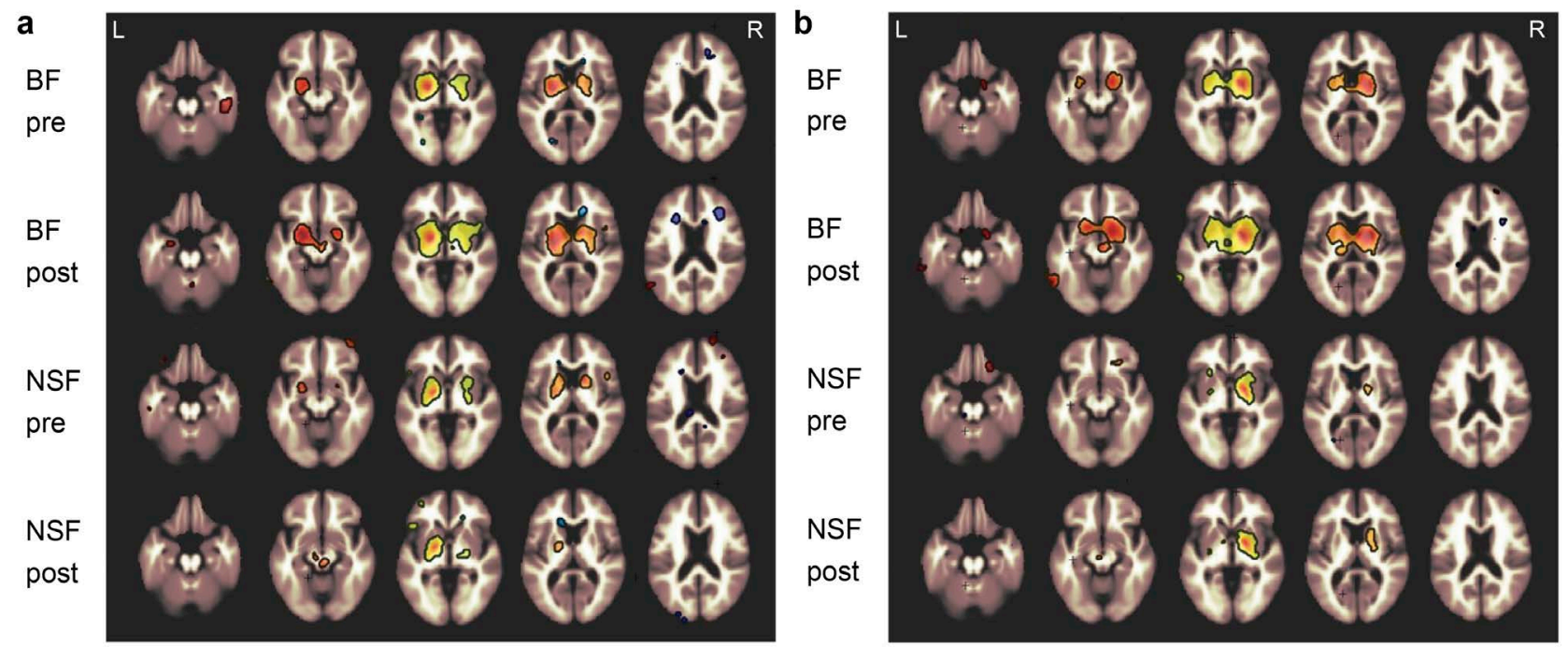

Figure 6. (a) Seed-to-voxel analysis of the pallidum (left). (b) Seed-to-voxel analysis of the pallidum (right). The activity of the pallidum was stronger in the BFs than in NSFs, and the reaction after makeup was stronger in the BFs. The pallidum is one of the subcortical structures of the brain and is part of the basal ganglia. It is divided into outer and inner segments, both of which contain large GABAergic projection neurons. L, left; R, right; BF, blind female; NSF, normal sighted female. 
In the other language inferior frontal gyrus (IFG) ( $L, R$ ), language posterior superior temporal gyrus, (pSTG) (L, R), visual medial, visual occipital, sensorymotor lateral $(\mathrm{L}, \mathrm{R})$, amygdala $(\mathrm{L}, \mathrm{R})$, caudate $(\mathrm{L}, \mathrm{R})$, hippocampus $(\mathrm{L}, \mathrm{R})$, putamen $(R, L)$, and thalamus $(L, R)$, no clear difference was noted in activity after makeup in the BFs or NSFs.

Excluding the visual lateral network, no difference was noted in the ROI-to-ROI population analysis between the BFs and NSFs, nor was there a difference due to the application of makeup.

In the subtraction analysis of the anatomical regions before and after the makeup task using SPM 12, there was no region in which the activity significantly increased after makeup in the BFs, whereas the activity was significantly higher before makeup in the right middle cingulate gyrus $(p=0.00047)$. Similarly, there was no region with an increase in the activity after makeup in the NSFs, and the activity was slightly higher before makeup in the right cerebral white matter and right anterior cingulate gyrus $(p=0.004)$. However, the activity was significantly stronger and more extensive in BFs than in sighted individuals before and after makeup on comparison of the BFs and NSFs, whereas there was no region in which the activity was significantly higher in the NSFs than in BFs.

\section{Discussion}

According to a 2015 estimate, there are 216 million persons with moderate to severe visual impairment worldwide-188 million with mild visual impairment and 36 million considered blind [10]. Persons developing visual impairment from a variety of causes receive good support, but considering that visual information accounts for a considerable portion of sensation, they may lead an inconvenient life.

The history of makeup in Japan is old. Cosmetics were initially transmitted from China in the late 6th century, and knowledge of makeup was established in the 17 th century. Daily makeup use among females is considered as grooming. Makeup not only to enables one's self-expression to others by creating an esthetic pretense, but also generates psychological effects such as relaxation and improved mood [11].

Ueno et al. investigated nerve activity using fMRI while subjects observed 144 pictures of the same face with and without makeup (48 pictures with makeup, 48 pictures without makeup, and 48 scrambled pictures). In the left orbitofrontal cortex (OFC) and right hippocampus, the activity was higher viewing faces with makeup than without, and activity in the right anterior cingulate cortex, left hippocampus, and left OFC increased in response to the enhanced facial beauty as a result of cosmetics use [12]. Sato et al. [1] analyzed the subjective happiness scale (SHS) score while using fMRI, and found a decreased fractional amplitude of low-frequency fluctuations (fALFF) in the right precuneus increased the SHS score, whereas the functional and effective connectivity in the right precuneus and right amygdala was positively correlated with the SHS score [1]. 
Although visually impaired persons cannot see their own face in a mirror, they recognize it by touch. In visually impaired females, the tactile sensation of the hands may replace the function of the eyes. Many studies and reviews using fMRI on brain function and plasticity of the visually impaired have been performed. Task studies on brain plasticity [13] [14] [15] [16] [17] report that in persons with early visual impairment, the visual area functions in processing tactile information (Braille reading) [18] [19], cerebellar activation during Braille reading [20], interpretation of words [21] [22] [23], and auditory processing [24].

Conversely, there are fewer reports on networks at rest. According to $\mathrm{Yu}$ et al., early blind subjects showed decreased functional connectivity between the left primary visual area and the bilateral supplementary motor area, pre- and postcentral gyri, superior parietal lobule, and the left superior and middle temporal gyri [25]. Burton et al. showed the functional connectivity between the visual cortex and region associated with cognitive control is more marked in BFs compared to NSFs [19]. No report was found using fMRI that focused on the activity in the accumbens and pallidum of the visually impaired at rest. Our study showed that higher activity of the accumbens and pallidum regions in BFs compared to NSFs is consistent with previous reports that these regions are involved in the reward system [26] [27], which is supported by the happiness scale per the questionnaire.

Our study captured fMRI changes in the brains of females with a large handicap (i.e., visual impairment) induced by applying makeup by themselves. Seven females who took a nationwide short course of blind makeup application participated in this study. As such, the sample size was small, but all subjects had bright expressions and were more positive about life by being able to apply their own makeup.

In visually impaired persons, we found no visual network to the opposite side was formed among large-scale networks in the brain at rest, which is a new discovery. In addition, reward system activity was significantly stronger in visually impaired persons than in sighted persons of the same age. The visual cortex of visually impaired persons is deeply incorporated into the functional system, instantiating temporary recall (realization) and caution regarding non-visual events. We found the interaction between the visual sensory area and non-sensory areas was decreased.

This is the first scientific report to show that cosmetics are also beneficial for blind people. With the advent of a globally aging society, this is a groundbreaking revelation which suggests that even without sight; it is possible for use of cosmetics to contribute to a long, proactive life.

In conclusion, our major findings were the network between the visual cortices was lacking on fMRI in visually impaired persons, and also, their reward systems, including the nucleus accumbens, pallidum, and hippocampus, were activated on fMRI during the image task at rest. This study suggests that based on their brain function, visually impaired females who apply makeup themselves as 
a fundamental daily activity can live fully and feel confident in society. For future studies, based on the landmark results of this study, the number of participants will be increased, and the findings will be tested in other types of disabilities.

\section{Acknowledgements}

We are grateful to the 7 blind subjects from across Japan who learned to perform blind makeup and participated in this study, and the 9 sighted persons for participating in this study. This study was supported by the TOKIWA Pharmaceutical Co., Ltd. H.M. and Y.M. are employees of TOKIWA Pharmaceutical Co., Ltd. The other authors report no conflict of interest.

\section{References}

[1] Sato, W., Kochiyama, T., Uono, S., Sawada, R., Kubota, Y., Yoshimura, S. and Toichi, M. (2019) Resting-State Neural Activity and Connectivity Associated with Subjective Happiness. Scientific Reports, 9, Article No. 12098. https://doi.org/10.1038/s41598-019-48510-9

[2] Rombouts, S.A., Barkhof, F., Goekoop, R., Stam, C.J. and Scheltens, P. (2005) Altered Resting State Networks in Mild Cognitive Impairment and Mild Alzheimer's Disease: An fMRI Study. Human Brain Mapping, 26, 231-239. https://doi.org/10.1002/hbm.20160

[3] Sheline, Y.I. and Raichle, M.E. (2013) Resting State Functional Connectivity in Preclinical Alzheimer's Disease. Biological Psychiatry, 74, 340-347. https://doi.org/10.1016/j.biopsych.2012.11.028

[4] Dennis, E.L. and Thompson, P.M. (2014) Functional Brain Connectivity Using fMRI in Aging and Alzheimer's Disease. Neuropsychology Review, 24, 49-62. https://doi.org/10.1007/s11065-014-9249-6

[5] Sheffield, J.M. and Barch, D.M. (2016) Cognition and Resting-State Functional Connectivity in Schizophrenia. Neuroscience \& Biobehavioral Reviews, 61, 108-120. https://doi.org/10.1016/j.neubiorev.2015.12.007

[6] Brakowski, J., Spinelli, S., Dörig, N., Bosch, O.G., Manoliu, A., Holtforth, M.G. and Seifritz, E. (2017) Resting State Brain Network Function in Major Depression-Depression Symptomatology, Antidepressant Treatment Effects, Future Research. Journal of Psychiatric Research, 92, 147-159. https://doi.org/10.1016/j.jpsychires.2017.04.007

[7] Jiang, L.W., Qian, R.B., Fu, X.M., Zhang, D., Peng, N., Niu, C.S. and Wang, Y.H. (2018) Altered Attention Networks and DMN in Refractory Epilepsy: A Resting-State Functional and Causal Connectivity Study. Epilepsy \& Behavior, 88, 81-86. https://doi.org/10.1016/j.yebeh.2018.06.045

[8] Ming, J., Thulborn, K.R. and Szlyk, J.P. (2012) Reproducibility of Activation Maps for Longitudinal Studies of Visual Function by Functional Magnetic Resonance Imaging. Investigative Ophthalmology \& Visual Science, 53, 6153-6163. https://doi.org/10.1167/iovs.11-8375

[9] Jaswal, R., Gohel, S., Biswal, B.B. and Alvarez, T.L. (2014) Task-Modulated Coactivation of Vergence Neural Substrates. Brain Connectivity, 4, 595-607. https://doi.org/10.1089/brain.2013.0216

[10] Burton, M.J., Faal, H.B., Ramke, J., Ravilla, T., Holland, P., Wang, N., West, S.K., 
Bourne, R.R.A., Congdon, N.G. and Foster, A. (2019) Announcing the Lancet Global Health Commission on Global Eye Health. The Lancet Global Health, 7, e1612-e1613. https://doi.org/10.1016/S2214-109X(19)30450-4

[11] Matsuoka, Y., Yoneda, K., Sadahira, C., Katsuura, J., Moriue, T. and Kubota, Y. (2006) Effects of Skin Care and Makeup under Instructions from Dermatologists on the Quality of Life of Female Patients with Acne Vulgaris. The Journal of Dermatology, 33, 745-752. https://doi.org/10.1111/j.1346-8138.2006.00174.x

[12] Ueno, A., Ito, A., Kawasaki, I., Kawachi, Y., Yoshida, K., Murakami, Y., Sakai, S., Iijima, T., Matsue, Y. and Fujii, T. (2014) Neural Activity Associated with Enhanced Facial Attractiveness by Cosmetics Use. Neuroscience Letters, 566, 142-146.

https://doi.org/10.1016/j.neulet.2014.02.047

[13] Park, H.J., Chun, J.W., Park, B., Park, H., Kim, J.I., Lee, J.D. and Kim, J.J. (2011) Activation of the Occipital Cortex and Deactivation of the Default Mode Network during Working Memory in the Early Blind. Journal of the International Neuropsychological Society, 17, 407-422. https://doi.org/10.1017/S1355617711000051

[14] Wang, X., Peelen, M.V., Han, Z., He, C., Caramazza, A. and Bi, Y. (2015) How Visual Is the Visual Cortex? Comparing Connectional and Functional Fingerprints between Congenitally Blind and Sighted Individuals. Journal of Neuroscience, 35, 12545-12559. https://doi.org/10.1523/JNEUROSCI.3914-14.2015

[15] Bedny, M. (2017) Evidence from Blindness for a Cognitively Pluripotent Cortex. Trends in Cognitive Sciences, 21, 637-648.

https://doi.org/10.1016/j.tics.2017.06.003

[16] Hoffmann, M.B., Thieme, H. and Ahmadi, K. (2017) Potential of fMRI for the Functional Assessment of the Pathological Visual System. Klinische Monatsblätter für Augenheilkunde, 234, 303-310. https://doi.org/10.1055/s-0043-103960

[17] van den Hurk, J., Van Baelen, M. and Op de Beeck, H.P. (2017) Development of Visual Category Selectivity in Ventral Visual Cortex Does Not Require Visual Experience. Proceedings of the National Academy of Sciences of the United States of America, 114, E4501. https://doi.org/10.1073/pnas.1612862114

[18] Sadato, N., Okada, T., Kubota, K. and Yonekura, Y. (2004) Tactile Discrimination Activates the Visual Cortex of the Recently Blind Naïve to Braille: A Functional Magnetic Resonance Imaging Study in Humans. Neuroscience Letters, 359, 49-52. https://doi.org/10.1016/j.neulet.2004.02.005

[19] Burton, H., Snyder, A.Z. and Raichle, M.E. (2014) Resting State Functional Connectivity in Early Blind Humans. Frontiers in Systems Neuroscience, 8, 51. https://doi.org/10.3389/fnsys.2014.00051

[20] Gizewski, E.R., Timmann, D. and Forsting, M. (2004) Specific Cerebellar Activation during Braille Reading in Blind Subjects. Human Brain Mapping, 22, 229-235. https://doi.org/10.1002/hbm.20031

[21] Burton, H., Snyder, A.Z., Diamond, J.B. and Raichle, M.E. (2002) Adaptive Changes in Early and Late Blind: a FMRI Study of Verb Generation to Heard Nouns. Journal of Neurophysiology, 88, 3359-3371. https://doi.org/10.1152/jn.00129.2002

[22] Neville, H. and Bavelier, D. (2002) Human Brain Plasticity: Evidence from Sensory Deprivation and Altered Language Experience. Progress in Brain Research, 138, 177-188. https://doi.org/10.1016/S0079-6123(02)38078-6

[23] Bedny, M., Richardson, H. and Saxe, R. (2015) "Visual" Cortex Responds to Spoken Language in Blind Children. Journal of Neuroscience, 35, 11674-11681. https://doi.org/10.1523/JNEUROSCI.0634-15.2015

[24] Hötting, K. and Röder, B. (2009) Auditory and Auditory-Tactile Processing in 
Congenitally Blind Humans. Hearing Research, 258, 165-174.

https://doi.org/10.1016/j.heares.2009.07.012

[25] Yu, C., Liu, Y., Li, J., Zhou, Y., Wang, K., Tian, L., Qin, W., Jiang, T. and Li, K. (2008) Altered Functional Connectivity of Primary Visual Cortex in Early Blindness. Human Brain Mapping, 29, 533-543. https://doi.org/10.1002/hbm.20420

[26] Smith, K.S., Tindell, A.J., Aldridge, J.W. and Berridge, K.C. (2009) Ventral Pallidum Roles in Reward and Motivation. Behavioural Brain Research, 196, 155-167. https://doi.org/10.1016/j.bbr.2008.09.038

[27] Floresco, S.B. (2015) The Nucleus Accumbens: An Interface between Cognition, Emotion, and Action. Annual Review of Psychology, 66, 25-52.

https://doi.org/10.1146/annurev-psych-010213-115159 